\title{
Análise motivacional, causal e funcional da brincadeira em duas espécies de roedores
}

\author{
Mauro Luís Vieira \\ Rodrigo Sartorio \\ Universidade Federal de Santa Catarina
}

\begin{abstract}
Resumo
A brincadeira é um fenômeno bastante comum em indivíduos jovens de diferentes espécies, principalmente mamíferos. O objetivo do presente artigo foi fazer uma revisão sobre pesquisas realizadas com roedores, mais especificamente hamsters dourados (Mesocricetus auratus) e ratos albinos (Rattus norvegicus). Esses animais apresentam os mais altos índices de complexidade de brincadeira e são os mais estudados em laboratório nessa área, entre os roedores. Com base nos artigos da literatura pesquisada, conclui-se que a brincadeira: a) é um sistema motivacional próprio que apresenta características que o diferencia de outros sistemas motivacionais; b) é modulada pelo sexo e idade do indivíduo e dos parceiros envolvidos na interação social; e c) o significado funcional pode estar relacionado com a preparação do indivíduo para viver em ambientes sociais e treinamento físico. Nesse sentido, a brincadeira pode ser relevante para se compreender aspectos do desenvolvimento comportamental e social.
\end{abstract}

Palavras-chave: Brincadeira, Roedores, Desenvolvimento, Motivação, Função.

\begin{abstract}
Motivational, causal and functional analysis of play behavior in two rodent species

Play behavior is a very frequent phenomenon in juvenile individuals of various species, mainly mammals. The purpose of the present article is to review the studies in rodents, more specifically in golden hamsters (Mesocricetus auratus) and albino rats (Rattus norvegicus). These animals show the highest indices of play complexity and are, among rodents, the animals most studied in the laboratory in this field of research. Through this review of the literature we conclude that play behavior: a) is a motivational system in its own right and possesses characteristics that differentiate it from other motivational systems; $b$ ) is modulated by the sex and age of individuals and of the partners involved in the social interaction; and c) has a functional significance that may be related to physical training and the preparation of the individuals for life in a social environment. In this way, play behavior can be relevant to the understanding of aspects of behavioral and social development.
\end{abstract}

Key words: Play behavior, Rodents, Development, Motivation, Function.

$\mathrm{A}$ brincadeira é um comportamento bastante freqüente na vida de animais jovens, principalmente mamíferos. O seu estudo pode ser considerado um meio pelo qual é possível compreender como ocorre o desenvolvimento comportamental e social. Apesar de termos diversos resultados de pesquisa, principalmente em relação aos fatores causais, ainda não é possível afirmar categoricamente para que serve a brincadeira e como ocorreu a sua evolução filogenética.
Apesar da aparente importância da brincadeira, não existe consenso sobre os méritos em considerá-la como uma categoria de comportamento útil e válida (Fagen, 1981). A dificuldade para estudar a brincadeira mantém muitos pesquisadores distantes desse tema (Byers, 1981). Em termos históricos, constata-se que houve grandes oscilações na publicação de artigos sobre brincadeira em roedores nas últimas três décadas. Através da análise da base de dados PsycINFO de 1970 até 2001, constata-se 
que as décadas de 80 e 90 foram bastante profícuas no estudo do comportamento lúdico em hamsters dourados e, principalmente, ratos albinos [esses roedores são os mais estudados em laboratório com relação ao estudo da brincadeira (Hole \& Einon, 1984; Vieira, 1995) e que apresentam os índices mais elevados de complexidade de brincadeira social, que inclui ataque, defesa, contra-ataque e perseguição, por exemplo (Iwaniuk, Nelson \& Pellis, 2001)].

Num total de 140 artigos, cerca de $90 \%$ destes foram publicados entre 1980 e 1999. Na década de 70 foram publicados apenas cinco artigos, segundo dados da PsycINFO. Em um outro estudo de revisão (Thor \& Holloway, 1984), restrito a ratos e apenas relacionado com a ontogenia (desenvolvimento ao longo da idade), os autores constataram que antes de 1970 apenas três artigos foram publicados sobre brincadeira social em ratos e nenhum artigo na década de 70 . Por outro lado, de 1980 a 1984, oito artigos foram publicados.

Através do levantamento realizado na base de dados PsycINFO, nota-se que nos anos de 2000 e 2001 a produção foi de apenas seis artigos publicados. Esses dados parecem indicar que o interesse dos pesquisadores em estudar a brincadeira em hamsters dourados e ratos albinos nos últimos dois anos é incipiente diante das dúvidas e indagações que ainda se tem sobre o referido comportamento.

Embora reconhecendo a dificuldade para fazer estudos científicos para determinar a relação de causa e efeito entre a brincadeira que ocorre durante o período juvenil e comportamentos de adultos, sugere-se que o estudo da brincadeira é um assunto relevante para se compreender o comportamento animal. Embora existam incongruências e problemas no estudo científico da brincadeira, este comportamento permanece um enigma biológico (nem sempre é possível identificar suas funções para o indivíduo ou para a espécie, embora os custos sejam mais visíveis) que pode e deve ser melhor investigado (Fagen, 1977; Martin \& Caro, 1985; Panksepp, 1981).

Portanto, através da presente revisão pretende-se identificar propriedades e características da brincadeira, além de identificar as causas internas (orgânicas e motivacionais) e externas (sociais) que controlam o início da brincadeira e modulam a continuidade e a interrupção do comportamento com base, principalmente, em pesquisas realizadas com hamsters dourados e ratos albinos. Esse empreendimento tem como finalidade apontar hipóteses para identificar as possíveis funções da interação lúdica no processo de desenvolvimento.

\section{Definição}

A facilidade com que uma pessoa identifica o comportamento de brincadeira é inversamente proporcional à sua definição em termos estruturais, ou seja, movimentos e ações envolvidos neste tipo de atividade. No entanto, embora não tenhamos uma definição precisa do que seja a brincadeira, podemos listar algumas de suas características. Diferentemente de outros comportamentos, tais como agressão e acasalamento, a brincadeira parece não ter uma importância biológica, prontamente percebida, justamente por não apresentar um ato consumatório (Bekoff \& Allen, 1998). O que se constata é a alternância de quem é dominado e dominante (Janus, 1987; Panksepp \& Beatty, 1980;). Além disso, os padrões motores se repetem com freqüência e são usados exageradamente (Fagen, 1981). Segundo Bekoff e Allen (1998), brincadeira é toda atividade motora que o indivíduo apresenta após o nascimento e que parece não ter um objetivo imediato, no qual padrões motores de outros contextos podem ser usados de forma modificada e alterada em termos de seqüência temporal.

Para Hole e Einon (1984), o critério mais adequado para considerar um comportamento como sendo brincadeira é quando o indivíduo usa padrões de comportamentos únicos. No caso específico da brincadeira social em roedores, embora possam ser usados comportamentos de outros contextos, tais como agressividade e interação sexual, eles são usados de forma exagerada ou incompleta. Esses comportamentos seriam uma forma de metacomunicação, informando ao parceiro que a interação a seguir é de "brincadeira" e não de "verdade".

A brincadeira apresenta diversos níveis de complexidade, indo desde simples corridas e saltos até interações sociais complexas e prolongadas (Fagen, 1981). Como não é um comportamento simples e unitário, a brincadeira geralmente é dividida em três subcategorias: 1) com objetos, 2) brincadeira envolvendo locomoção e, 3) brincadeira social (Burghardt, 1984; Hole \& Einon, 1984; Martin \& Caro, 1985).

\section{Sistema motivacional}

De um modo geral, pode-se dizer que a brincadeira, além de ter características específicas, como foi visto anteriormente, compartilha propriedades em comum com outros sistemas motivacionais indispensáveis para a sobrevivência do indivíduo, tais como o comer e o beber. Por exemplo, é bem estabelecido na literatura que após um período de isolamento social, o comportamento de brincadeira aumenta significativamente (Panksepp \& Beatty, 1980; Thor \& Holloway, 1984). Além disso, ocorre o processo de saciação. Em ratos, constatou-se que a freqüência da brincadeira decai com o passar do tempo (Hole, 1991).

Através de um experimento realizado com o hamster dourado, Vieira e Otta (1997) constataram que após períodos de 8, 24 e 48 horas de isolamento social, o tempo despendido em brincadeira foi proporcional ao período em que os animais ficaram sozinhos. No entanto, não houve diferença entre os grupos com relação ao tempo 
despendido em contato físico. Esse resultado indica que os efeitos do isolamento social não foram os mesmos para as diferentes categorias de comportamento. Por outro lado, constatou-se que houve decréscimo gradativo no tempo de brincadeira ao longo da sessão experimental, em todos os grupos, o que indica um processo de saciação. Esse mesmo resultado foi obtido tanto para animais privados como não privados (Vieira, 2001). No caso de animais não privados, apenas houve a troca de parceiros, indicando que o efeito de novidade também é uma variável que tem influência sobre a interação dos hamsters dourados, $o$ que talvez possa ser generalizado para outras espécies.

Por outro lado, ratos aprendem o caminho de um labirinto quando a recompensa é a oportunidade para brincar com um companheiro (Humphreys \& Einon, 1981). Outro resultado obtido nesse estudo foi que os ratos preferiam brincar com parceiros que estavam livres do que confinados. No entanto, esse resultado por si só não comprova que a escolha esteja relacionada com a brincadeira. Talvez a simples interação física fosse suficiente. Essa hipótese foi testada. Os autores manipularam o comportamento social do parceiro do rato com auxílio de drogas. Formaram-se duplas de ratos jovens e um dos parceiros tinha seu comportamento alterado através de drogas. Em um dos grupos, o parceiro recebeu doses de clorpromazina e em outro de anfetamina. Essas drogas diminuem a freqüência de brincadeira, sendo que a primeira é depressiva e pode causar aumento na freqüência de comportamentos amigáveis (não incluindo a brincadeira), como, por exemplo, cheirar o companheiro, mover-se em sua direção ou simplesmente manter-se em contato físico com ele. A anfetamina causa hiperatividade. Através dos resultados, constatou-se que os animais tratados com drogas foram menos preferidos, em comparação com grupos controles, em que os animais eram expostos a um parceiro normal. Isto sugere que o comportamento de brincar foi importante para determinar a atratividade animal que estava sendo testada. Quando as solicitações para brincar não são respondidas, ratos acabam diminuindo marcadamente tais comportamentos (Thor \& Holloway, 1983). Portanto, percebe-se que a brincadeira pode ser um evento reforçador e estimulante para o animal.

Todos esses dados sugerem a existência de um processo regulador, indicando que a brincadeira pode constituir-se em um sistema motivacional próprio, que é controlado por diversos fatores, como veremos a seguir.

\section{Fatores causais}

Além da definição, outro aspecto que chama a atenção dos pesquisadores que investigam a brincadeira são os fatores que regulam o aparecimento e a duração dessa atividade. Em termos práticos, podemos dividir esses fa- tores em dois grandes grupos: internos (endógenos e motivacionais) e externos (sociais).

Com relação aos aspectos internos, nota-se que existem diferenças marcantes quanto ao sexo do indivíduo e a brincadeira. Meaney, Stewart e Beatty (1985) fizeram uma revisão dos principais resultados de pesquisas realizadas com crianças, macacos, babuínos, chimpanzés, sagüis, cães, coiotes, lobos, gatos, ratos, hamsters, gerbilos e ovelhas. Examinando-se os dados apresentados conclui-se que, na maioria das espécies estudadas até hoje, o macho brinca mais que a fêmea. Apenas em lobos, cães, coiotes, gatos e gerbilos a fêmea brinca tanto quanto o macho. Em nenhum dos estudos revistos a fêmea despende mais tempo em brincadeira do que o filhote macho.

No caso específico de roedores, ratos machos iniciam mais comportamentos de brincadeira do que fêmeas (Meaney \& Stewart, 1981; Poole \& Fish, 1976; Thor \& Holloway, 1983). Por outro lado, fêmeas interrompem mais frequentemente um episódio de brincadeira do que machos (Meaney et al., 1985) e os eventos de interação lúdica entre elas tendem a ser mais curtos (Hole, 1988).

A exposição a andrógenos durante períodos precoces do desenvolvimento cerebral influencia a freqüência da brincadeira durante o período juvenil. Observou-se que a administração de testosterona (androgênio primário), ainda no período neonatal, masculiniza o comportamento social de ratas fêmeas - incluindo a brincadeira (Olioff \& Stewart, 1978; Pellis \& McKenna, 1992; Pellis, Pellis \& Kolb, 1992; Pellis, Pellis \& McKenna, 1994). Ward e Stehm (1991) comprovaram empiricamente que o estresse pré-natal da mãe provocou diminuição da brincadeira em ratos machos. Portanto, parece que a diferenciação sexual da brincadeira é devida, em parte, a ação hormonal que ocorre mesmo antes do nascimento (Meaney, 1988).

Além disso, outro fator que interfere decisivamente no comportamento lúdico é a idade do indivíduo. Já é bem documentada na literatura que a brincadeira é uma atividade característica de animais jovens que estão em desenvolvimento. No rato albino a brincadeira aparece em torno do $15^{\circ}$ dia de idade, aumenta de freqüência nos dias posteriores e atinge um pico por volta do $36^{\circ}$ dia, decaindo posteriormente (Meaney \& Stewart, 1981). No hamster dourado também pode ser observada uma tendência desse tipo, embora o período de pico seja um pouco diferente, ou seja, entre 25 e 35 dias de idade (Pellis \& Pellis, 1988a). A maturidade sexual do hamster inicia-se justamente em torno do $35^{\circ}$ dia de vida. Após esse período o nível de tolerância entre os indivíduos decai e, conseqüentemente, diminui também a taxa de contato fisico e outras formas de interação social, como a brincadeira. 
Por outro lado, os fatores externos (sociais) também podem nos ajudar a explicar por que machos e fêmeas brincam diferentemente. $\mathrm{O}$ sexo do parceiro pode ser uma variável importante. Em hamsters dourados, díades de sexos diferentes despendem significativamente mais tempo em contato físico, mas menos tempo em brincadeira, quando comparadas com díades de mesmo sexo (Guerra, Vieira, Takase \& Gasparetto, 1992). Em ratos albinos notou-se que a brincadeira ocorria com maior freqüência entre indivíduos de mesmo sexo (Meaney \& Stewart, 1981). A compatibilidade comportamental é uma das possíveis explicações para esses resultados. O comportamento do parceiro de mesmo sexo seria mais atraente do aquele do sexo oposto. Essa hipótese também poderia ser extrapolada para outras características do parceiro tais como idade?

De um modo geral, pode-se dizer que o comportamento de brincadeira decai com a idade, como foi visto anteriormente. Isso não quer dizer que o adulto não se envolva em brincadeiras, mas a freqüência é baixa, em comparação com os jovens. Nesse sentido, atividades ou ações sem benefício visível, como é caso da brincadeira, seriam pouco freqüentes entre animais que já passaram pelo período juvenil. Além disso, há perigo potencial para os indivíduos envolvidos na interação social, podendo mais facilmente resultar em agressão do que entre indivíduos jovens. O adulto, portanto, não é um bom parceiro para o indivíduo jovem, conforme foi constatado por experimentos, como veremos a seguir.

Através de pesquisa em que foi utilizado o hamster dourado como sujeito experimental, constatou-se que a mãe não consegue suprir totalmente a falta de um companheiro de ninhada para seu filhote (Vieira \& Guerra, 2001). Inicialmente foram criados três grupos. Em um deles o filhote ficava sozinho durante 48 horas. Em outro grupo o filhote ficava com a mãe e, no terceiro grupo, o filhote ficava com um parceiro de mesma idade. Na situação teste, em que se agrupavam dois animais provenientes da mesma situação descrita acima, constatou-se que o tempo despendido em contato físico e em brincadeira foi maior entre os animais que estavam isolados, significativamente menor entre os animais do grupo controle (filhote-filhote) e intermediário no grupo em que o filhote ficou com a mãe. No entanto, quando se analisou de forma mais detalhada a natureza da interação social entre os filhotes, constatou-se que o comportamento de filhotes que ficaram com a mãe foi mais parecido com o comportamento dos filhotes que haviam sido isolados totalmente de interações sociais.

Pode-se dizer que a permanência apenas com a mãe parece significar uma forma de privar os filhotes dos benefícios da interação social entre parceiros de mesma idade, principalmente em relação à brincadeira. Portanto, sugere-se que, em condições normais, talvez exista uma tendência para selecionar parceiros de mesma idade, peso e força física para brincar.

Com base no que foi apresentado até agora na presente revisão pode-se concluir que hamsters dourados e ratos albinos jovens apresentam forte motivação para brincar. No entanto, a modulação desse comportamento é realizada por variáveis tais como o sexo e a idade dos indivíduos e dos parceiros. Depois de identificar as propriedades da brincadeira e determinar os fatores causais desse comportamento, o próximo passo é saber para que serve a brincadeira.

\section{Significado funcional}

Através da análise da distribuição filogenética da brincadeira, constata-se que este comportamento está relacionado com a complexidade cerebral, comportamentos generalizados e o papel que a aprendizagem desempenha no desenvolvimento do comportamento. Por outro lado, a ausência de brincadeira em répteis pode ser explicada em termos de dispêndio de energia (Burghardt, 1984). A limitação fisiológica, que não permite o armazenamento de energia, e o grande risco de predação a que estão submetidas diversas espécies de répteis, contribuíram para que a brincadeira não fizesse parte do repertório comportamental desses animais.

A partir desse argumento, pode-se deduzir que os custos da brincadeira são provavelmente elevados e que o retorno, em termos de benefícios, também é considerável. Até a década de 60 concordava-se que a brincadeira consumia grande parte do tempo e energia do animal e que poderia envolver riscos físicos. Sendo assim, deveria ter importância adaptativa crucial no desenvolvimento e sobrevivência da espécie. No entanto, até hoje, ainda não se têm provas empíricas de que o tempo e energia despendidos durante a brincadeira são realmente elevados e que tal comportamento é essencial para o desenvolvimento (Martin \& Caro, 1985).

Estudos realizados com várias espécies mostraram que o tempo gasto em brincadeira não ultrapassa $10 \%$ do orçamento de atividades (Martin, 1984; Martin \& Caro, 1985; Miller \& Byers, 1991). No caso específico de roedores, Siviy e Atrens (1992), constataram que, em ratos albinos, a brincadeira consome entre 2 e $3 \%$ do orçamento energético diário.

Diferentes procedimentos metodológicos têm sido utilizados para avaliar o custo energético da brincadeira. Por exemplo, no estudo de Siviy e Atrens (1992), os ratos foram colocados para brincar numa câmara cilíndrica, medindo 20 centímetros de diâmetro. Através de equipamentos computadorizados mediu-se o consumo de oxigênio e a produção de dióxido de carbono, quantificandose a partir desses dados o gasto de energia. 
Outra forma que tem sido utilizada para avaliar os custos da brincadeira é a quantificação da porcentagem do tempo diário despendido na atividade. Em ratos este valor chega a 3\% (Thiels, Alberts \& Cramer, 1990). Os autores filmaram os animais durante períodos de 24 horas, do $12^{\circ}$ até o $34^{\circ}$ dia de vida dos filhotes. Além da brincadeira, constatou-se que os ratos, nos últimos dias da observação, passaram cerca de 55\% do tempo dormindo e $10 \%$ do tempo comendo ou bebendo água.

Com base nos dados apresentados acima pode-se sugerir que em função do tempo despendido em brincadeira e dos custos envolvidos nessa atividade, os efeitos seriam mínimos para que a brincadeira pudesse ser mantida pela seleção natural (Martin \& Caro, 1985). Esses autores também argumentam que o comportamento lúdico pode ser adaptativo devido aos seus efeitos imediatos ou, em curto prazo, e pode não ter efeitos de longo prazo consistentes, importantes e previsíveis.

Por outro lado, segundo Fagen (1981), os benefícios da brincadeira podem ser enquadrados em seis hipóteses: 1) treinamento físico; 2) desenvolvimento de habilidades sociais; 3) regulação da taxa de desenvolvimento; 4) competição intra-específica; 5) promoção de vínculos e coesão social; e 6) aprendizagem de informações específicas. Para facilitar a análise funcional, agruparemos estas hipóteses em dois grupos: (1) treinamento de habilidades motoras e (2) desenvolvimento de habilidades sociais, envolvendo tanto a competição como a coesão social.

Com relação à hipótese do desenvolvimento de habilidades sociais, a brincadeira pode servir para desenvolver e manter vínculos sociais. A interação lúdica pode facilitar a manutenção de laços sociais e a integração na estrutura social do grupo, como é caso do rato albino (Meaney et al., 1985; Panksepp, 1981). Pellis e Pellis (1991) constataram que em ratos albinos, desde o desmame até o início da maturidade sexual (período em que a brincadeira é mais freqüente), a freqüência com que um parceiro fica sobre o outro ou lança ataques e contra-ataques, torna-se progressivamente assimétrica, ou seja, começa a ocorrer dominância de um animal sobre o outro. Contudo, não foram os animais que apresentaram as maiores taxas de início de brincadeira que se tornaram dominantes em idades mais avançadas. Parece que o padrão de comportamento de ratos subordinados, mesmo durante as interações lúdicas, tem como função a manutenção de 'laços de amizade', permitindo a coexistência pacífica em colônias (Pellis \& Pellis, 1992).

Além disso, durante a brincadeira social também ocorre o treinamento de habilidades motoras. No caso específico do hamster dourado, a função da brincadeira pode estar relacionada com o treinamento motor e o aprendizado sobre interações afiliativas e sexuais. No primeiro caso, durante o período em que o filhote fica junto com a mãe e os irmãos, a brincadeira pode ser uma maneira pela qual o hamster desenvolve sua aptidão física, sem, no entanto, se dispersar da ninhada, já que vive em tocas, em situação natural. Quanto aos efeitos anatômicos e fisiológicos, o treinamento motor pode: (1) desenvolver e fortalecer ossos e músculos, além de (2) aumentar a eficiência da capacidade cardiopulmonar (Bekoff, 1988).

A brincadeira turbulenta pode aumentar a habilidade de um animal para enfrentar as flutuações diárias e sazonais da temperatura ambiente (Siviy \& Atrens, 1992). Com efeito, um animal que se exercita fisicamente poderia estar mais bem preparado para enfrentar quedas de temperatura.

Durante as interações lúdicas, constata-se que as mordidelas ou simples contato oral são dirigidos para a região das bolsas bucais do companheiro, enquanto nos encontros agressivos as mordidelas são dirigidas para a parte traseira do oponente, tanto em hamsters dourados (Pellis \& Pellis, 1988b) como em ratos albinos (Pellis \& Pellis, 1987). Estas constatações sugerem que a brincadeira juvenil está mais relacionada com as interações sociais e sexuais do que com interações agonísticas.

Em ratos albinos, pesquisa recente tem mostrado que a brincadeira é indispensável para o desenvolvimento adequado de enfrentamento relacionado com o ambiente social (van den Berg, Hol, Van Ree \& Spruijt, 1999). Nesse estudo, ratos jovens foram privados de interações sociais durante a quarta e quinta semanas de vida, que é o período em que a brincadeira social no rato atinge o pico (Meaney \& Stewart, 1981). Na sexta semana os animais eram testados. Através dos resultados os autores constataram que a privação social resultou no decréscimo da atividade social (incluindo o comportamento sexual), mas não afetou a atividade locomotora ou o desempenho do animal no teste de cruz elevada. Os ratos privados também não apresentaram prontamente postura de submissão ou imobilidade em resposta a um residente, além de apresentarem maiores concentrações de corticosteróide e adrenalina, quando comparados com animais não isolados. Em outro estudo também com ratos albinos, constatou-se que não existe diferença na agressão intra-específica e no comportamento predatório entre animais criados em isolamento social e junto com companheiros (Potegal \& Einon, 1989). A interação lúdica poderia ser uma oportunidade em que os indivíduos aprendem a inibir a agressividade.

Em termos metodológicos existem questionamentos sobre a utilização do isolamento social como meio para 
comprovar a importância da brincadeira. $\mathrm{O}$ animal privado de interações sociais não fica apenas privado de brincadeira, mas outros comportamentos também são alterados quando ele está sozinho (Martin \& Caro, 1985). Contudo, foram realizados experimentos em que foi possível relacionar a falta de brincadeira com alterações de comportamento. Os efeitos deletérios do isolamento social em ratos albinos criados sozinhos entre 25 e 45 dias de idade foram minimizados se os animais pudessem brincar com um parceiro durante uma hora por dia (Einon, Morgan $\&$ Kibbler, 1978). Em outro experimento, que já foi citado anteriormente (Vieira \& Guerra, 2001), a privação seletiva de contatos sociais indicou que filhotes de hamsters dourados criados apenas pela mãe (que raramente brinca) apresentaram alguns comportamentos semelhantes a filhotes totalmente isolados.

Com base nas colocações anteriores, evidencia-se claramente a noção de que as experiências que ocorrem nos estágios iniciais da vida, incluindo nesse caso a brincadeira, teriam como um dos objetivos preparar o indivíduo para desempenhar comportamentos que serão necessários para a sobrevivência como adulto. No entanto, o indivíduo jovem apresenta necessidades e motivações que são específicas ao seu estágio de vida. A seleção natural atua não somente na vida adulta dos animais, mas também ao longo de todo o processo ontogenético. Um indivíduo deve se adaptar às condições ecológicas ou ambientais que são apresentadas a ele em cada estágio da vida (Oppenheim, 1980; Williams, 1966). Nesse sentido, como a brincadeira é um comportamento mais característico de animais durante o período juvenil, devemos também buscar relações entre esse comportamento e as necessidades do indivíduo jovem.

Mais recentemente, autores têm proposto uma nova estrutura conceitual para se investigar as funções da brincadeira. Spinka, Newberry e Bekoff (2001) propõem que a brincadeira capacita aos animais desenvolver versatilidade de movimentos e respostas emocionais emitidas para se recuperar de situações aversivas inesperadas, nas quais os animais experienciam perda de controle (o que os autores chamam de "treinamento para o inesperado"). Durante a brincadeira os animais criam e buscam situações inesperadas, relaxam o controle sobre seus movimentos e ativamente se colocam em situações e posições de desvantagem. Nesse sentido, a brincadeira constitui-se de seqüências de comportamentos nas quais os indivíduos mudam rapidamente entre movimentos bem definidos e controlados similar àqueles comportamentos "sérios" apresentados em outros contextos e movimentos de auto-desajustamento que resultam na perda temporária do controle. Como conseqüência dessa situação, surgiria um estado emocional complexo denominado pelos autores de "having fun", o qual teria exigências cognitivas, repercussões filogenéticas e restrições ontogenéticas sobre a brincadeira, além de respostas neuroendocrinológicas correlacionadas.

Com base nessa estrutura teórica, os autores apresentam 24 predições que são examinadas com base em dados empíricos e contrastando-as com predições de hipóteses alternativas. Ao final do artigo, Spinka et al. (2001) concluem que a hipótese do "treinamento para o inesperado" pode explicar alguns dos quebra-cabeças relacionados com aspectos motores, estruturais, motivacionais, emocionais, cognitivos, sociais, ontogenéticos e filogenéticos da brincadeira.

Como se vê, a proposta é nova e audaciosa. Embora esteja amparada em resultados de experimentos, necessita maiores avaliações pois, como mencionado pelos autores, as evidências são fragmentadas, a revisão da literatura sobre brincadeira não está completa e nem sempre foi possível comparar dados de diferentes pesquisas usando critérios uniformes, devido a variações metodológicas.

\section{Conclusão}

A brincadeira é um fenômeno comportamental complexo e, apesar das pesquisas já realizadas, ainda não temos teorias consistentes que nos permitam afirmar com relativa segurança o significado funcional de tal comportamento. Pesquisas atuais têm procurado identificar as origens evolutivas (Bekoff \& Byers, 1998; Iwaniuk et al., 2001; Pellis \& Iwaniuk, 1999, 2000) e as funções ontogenéticas (Spinka et al., 2001; van der Berg et al., 1999) da brincadeira em animais de um modo geral e, de roedores, de modo particular.

Devido à complexidade do comportamento, começando pela sua definição, é necessário criar estratégias para desvendar um dos grandes enigmas do comportamento animal, que é o significado funcional da brincadeira (Caro, 1990; Martin \& Caro, 1985). Uma das estratégias é a análise comparativa. A comparação entre espécies permite buscar critérios que possam ser uniformes na definição da categoria comportamental. Existem cerca de 3000 espécies de roedores conhecidas, mas o rato albino é o único cujo comportamento lúdico foi estudado com certa profundidade (Hole \& Einon, 1984). Problemas de definição têm levado alguns autores a separar comportamentos que são similares, como é o caso da brincadeira em hamsters dourado e ratos albinos (Hole \& Einon, 1984).

Como foi colocado na introdução desse artigo, existem muitos problemas em relação ao estudo da brincadeira. No entanto, isso não quer dizer que devemos deixar de lado esse conceito. Através da presente revisão procurou-se mostrar que a brincadeira em hamsters dourados e ratos albinos tem 
características específicas que a distingue de outras categorias de comportamento. Sugere-se que essa conclusão também pode ser extrapolada para outras espécies. Por outro lado, devido aos problemas inerentes aos estudos relacionados a esta área, é necessário criar estratégias que nos permitam afirmar com relativa segurança $o$ que é e para que serve a brincadeira, além de identificar os fatores causais desse comportamento.

Uma das estratégias para testar o significado funcional da brincadeira é o isolamento social. No entanto, existem problemas com a utilização desse paradigma, pois além do contato e da interação física, outras modalidades sensoriais são afetadas (Martin e Caro, 1985). De qualquer forma, os animais podem sofrer privação seletiva, sendo colocado em companhia de adultos, que não brincam ou brincam muito pouco ou são colocados com parceiros que tem seu comportamento alterado em função da injeção de drogas (que torna o animal hiperativo ou hipoativo). Nesses casos, procura-se eliminar a possibilidade de ocorrer brincadeira, sem que, no entanto, o animal fique privado de contatos físicos. O controle do tempo que os animais dispõem para brincar e do período de vida em que ocorre o isolamento social também podem fornecer indicadores do que acontece, a curto e, em longo prazo, quando a oportunidade para brincar é restrita.

Por outro lado, como a brincadeira é uma categoria comportamental complexa, é necessário procurar situações que possam facilitar a investigação do fenômeno. A brincadeira em roedores é eminentemente social e a seqüência de comportamentos envolvida nessa atividade é bem menor quando comparada a de primatas, por exemplo. Essa simplificação pode trazer vantagens para o pesquisador quando este se propõe a investigar com detalhes a natureza do comportamento. Além disso, a possibilidade de controle de variáveis e o período curto de vida dos roedores utilizados em laboratório talvez sejam fatores decisivos que nos permitem fazer descobertas de valor heurístico para compreender o que é, para que serve e o que modula a brincadeira em animais.

Como este artigo foi basicamente sobre a brincadeira em roedores (hamsters dourados e ratos albinos), sugere-se que os próximos artigos teóricos de revisão versem sobre o que é possível encontrar de semelhante ou de diferente entre o comportamento lúdico de diferentes ordens, tais como roedores e primatas (humanos e não-humanos).

\section{Referências}

Bekoff, M. (1988). Motor training and physical fitness: possible short and long-term influences on the development of individual differences in behavior. Developmental Psychobiology, 21, 601-612.

Bekoff, M., \& Allen, C. (1998). Intentional communication in social play: how and why animals negotiate and agree to play. In M. Bekoff \& J. A.
Byers (Orgs.), Play: Evolutionary, comparative and ecological perspectives (pp. 97-114). Cambridge: Cambridge University Press.

Bekoff, M., \& Byers, J. A. (Orgs.). (1998). Play: Evolutionary, comparative and ecological perspectives. Cambridge: Cambridge University Press.

Burghardt, G. M. (1984). On the origins of play. In P. K. Smith (Org.), Play in animals and humans (pp. 5-41). Oxford: Basil Blackwell..

Byers, J. (1981). The significance of play. Science, 212, 1493-1494.

Caro, T. M. (1990). Le jeu chez les animaux. La Recherche, 222, 740-747.

Einon, D. F., Morgan, M. J., \& Kibbler, C. C. (1978). Brief periods of socialization and later behavior in the rat. Developmental Psychobiology, 11, 213-225.

Fagen, R. M. (1977). Selection for optimal age-dependent schedules of play behavior. American Naturalist, 111, 395-414.

Fagen, R. M. (1981). Animal Play Behavior. Nova York: Oxford University Press.

Guerra, R. F., Vieira, M. L., Takase, E., \& Gasparetto, S. (1992). Sex differences in the play fighting activity in golden hamster infants. Physiology \& Behavior, 52, 1-5.

Hole, G. (1988). Temporal feature of social play in the laboratory rat. Ethology, 78, 1-20.

Hole, G. (1991). The effects of social deprivation on levels of social play in the laboratory rat (Rattus norvegicus). Behavioral Processes, 25 , 41-53.

Hole, G., \& Einon, D. F. (1984). Play in rodents. In P. K. Smith, (Org.), Play in Animals and Humans (pp. 95-117). Oxford: Basil Blackwell.

Humphreys, A. P., \& Einon, D. F. (1981). Play as a reinforcer for mazelearning in juvenile rats. Animal Behaviour, 29, 259-270.

Iwaniuk, A. N., Nelson, J. E., \& Pellis, S. M. (2001). Do big-brained animals play more? Comparative analyses of play and relative brain size in mammals. Journal of Comparative Psychology, 115, 29-41.

Janus, K. (1987). Early separations of young rats from the mother and the development of play fighting. Physiology \& Behavior, 39, 471-476.

Martin, P. (1984). The time and energy costs of play behaviour in the cat. Zeitchrift fur Tierpsychologie, 64, 298-312.

Martin, P., \& Caro, T. M. (1985). On the functions of play and its role in behavioral developmental. Advances in the Study of Behavior, 15, 59-103.

Meaney, M. J. (1988). The sexual differention of social play. Trends in Neuroscience, 11, 54-58.

Meaney, M. J., \& Stewart, J. (1981). A descriptive study of social development in the rat (Rattus norvegicus). Animal Behaviour, 29, $34-45$.

Meaney, M. J., Stewart, J., \& Beatty, W. W. (1985). Sex differences in social play: the socialization of sex roles. Advances in the Study of Behavior, 15, 1-56.

Miller, M. N., \& Byers, J. A. (1991). Energetic cost of locomotor play in pronghorn faws. Animal Behaviour, 41, 1007-1013.

Olioff, M., \& Stewart, J. (1978). Sex differences in the play behavior of prepubescent rats. Physiology \& Behavior, 20, 113-115.

Oppenheim, R. W. (1980). Metamorphosis and adaptation in the behavior of developing organism. Developmental Psychobiology, 13, 353-356.

Panksepp, J. (1981). The ontogeny of play in rats. Developmental Psychobiology, 14, 327-332.

Panksepp, J., \& Beatty, W. W. (1980). Social deprivation and play in rats. Behavioral and Neural Biology, 30, 197-206.

Pellis, S. M., \& Iwaniuk, A. N. (1999). The roles of phylogeny and sociality in the evolution of social play in muroid rodents. Animal Behaviour, 58, 361-373.

Pellis, S. M., \& Iwaniuk, A. N. (2000). Comparative analyses of the role of postnatal development on the expression of play fighting. Development Psychobiology, 36, 136-147. 
Pellis, S. M., \& McKenna, M. M. (1992). Intrinsic and extrinsic influences on play fighting in rats: effects of dominance, partner's playfulness, temperament and neonatal exposure to testosterone propionate. Behavioural Brain Research, 50, 135-145.

Pellis, S. M., \& Pellis, V. C. (1987). Play-fighting differ from serious fighting in both target of attack and tactics of fighting in the laboratory rat (Rattus norvegicus). Aggressive Behavior, 13, 227-242.

Pellis, S. M., \& Pellis, V. C. (1988a). Play-fighting in the syrian golden hamster Mesocricetus auratus water-house, and its relationship to serious fighting during post-weaning development. Developmental Psycobiology, 21, 323-337.

Pellis, S. M., \& Pellis, V. C (1988b). Identification of the possible origin of the body target that differentiates play-fighting from serious fighting in syrian golden hamster (Mesocricetus auratus). Aggressive Behavior, 14, 437-449.

Pellis, S. M., \& Pellis, V. C. (1991). Role reversal changes during the ontogeny of play fighting in male rats: attack versus defense. Aggressive Behavior, 17, 179-189.

Pellis, S. M., \& Pellis, V. C. (1992). Juvenilized play fighting in subordinate male rats. Agressive Behavior, 18, 449-457.

Pellis, S. M., Pellis, V. C., \& Kolb, B. (1992). Neonatal testosterone augmentation increases juvenile play fighting but does not influence the adult dominance relationships of male rats. Aggressive behavior, $18,437-447$.

Pellis, S. M., Pellis, V. C., \& McKenna, M. M. (1994). Feminine dimension in the play fighting of rats (Rattus norvegicus) and its defeminization neonatally by androgens. Journal of Comparative Psychology, 108, 68-73.

Poole, T. B., \& Fish, J. (1976). An investigation of individual, age, and sexual differences in the play of Rattus norvegicus (Mammalia: Rodentia). Journal of the Zoological Society, London, 179, 249-260.

Potegal, M., \& Einon, D. (1989). Aggressive behaviors in adult rats deprived of playfighting experience as juveniles. Developmental Psychobiology, $22,159-172$.
Siviy, S. M., \& Atrens, D. M. (1992). The energetic costs of rough-andtumble play in the juvenile rat. Developmental Psychobiology, 25, 137-148.

Spinka, M., Newberry, R. C., \& Bekoff, M. (2001). Mammalian play: training for the unexpected. Quarterly Review of Biology, 76, 141-168.

Thiels, E., Alberts, J. R., \& Cramer, C. P. (1990). Weaning in rats: II. Pup behavior patterns. Developmental Psychobiology, 23, 495-510.

Thor, H. D., \& Holloway, W. R., Jr. (1983). Play-solicitation behavior in juvenile male and female rats. Animal Learning \& Behavior, 11, 173178.

Thor, H. D., \& Holloway, W. R., Jr. (1984). Developmental analysis of social play behavior in juvenile rats. Bulletin of the Psychonomic Society, 22, 587-590

van der Berg, C. L., Hol, T., Van Ree, J. M., \& Spruijt, B. M. (1999). Play is indispensable for an adequate development of coping with social challenges in the rat. Development Psychobiology, 34, 129-138.

Vieira, M. L. (1995). A brincadeira em hamsters dourados (Mesocricetus auratus): influências do contexto social imediato e da privação soci$a l$. Tese de doutoramento não-publicada. Universidade de São Paulo, São Paulo.

Vieira, M. L. (2001). Semelhanças e diferenças na estrutura da brincadeira social de hamsters dourados (Mesocricetus auratus) isolados e não isolados. Biotemas, 14, 19-36.

Vieira, M. L., \& Guerra, R. F. (2001). Effects of social deprivation and interactions with play-unresponsive mothers on play fighting of juvenile golden hamsters. Ciência e Cultura, 53, 11-16.

Vieira, M. L., \& Otta, E. (1997). Efeitos de diferentes períodos de privação social sobre a brincadeira de hamsters dourados (Mesocricetus auratus). Biotemas, 10, 29-46.

Ward, I. L., \& Stehm, K. (1991). Prenatal stress feminizes juvenile play patterns in male rats. Physiology \& Behavior, 50, 601-605.

Williams, G. (1966). Adaptation and natural selection. Princeton: Princeton University Press.

Mauro Luís Vieira, doutor em Psicologia Experimental pela Universidade de São Paulo, SP e pósdoutorado pela Dalhousie University, DAL (Canadá), é professor do Departamento de Psicologia da Universidade Federal de Santa Catarina, SC.

Rodrigo Sartorio, mestre em Neurociências pela Universidade Federal de Santa Catarina, SC, é atualmente doutorando em Psicobiologia na Universidade Federal do Rio Grande do Norte, RN. Endereço para correspondência: Universidade Federal de Santa Catarina, Departamento de Psicologia, Campus Universitário - Trindade, 88049-900, Florianópolis, SC. Telefone (048)331.9639. Email:mvieira@cfh.ufsc.br. 